\title{
Contribution à la dépollution d'un rejet textile par adsorption sur un coagulant à base du mélange fer/aluminium préparé par électrocoagulation
}

\author{
Fatiha ZIDANE ${ }^{1 *}$, Noureddine KABA ${ }^{1}$, Jalila BENSAID ${ }^{1}$, Jean François BLAIS ${ }^{2}$, \\ Patrick DROGUI ${ }^{2}$, Adil RHAZZAR ${ }^{1}$, Brahim LEKHLIF ${ }^{3}$ et Bahae BENABDENBI ${ }^{1}$ \\ ${ }^{1}$ Laboratoire Science de l'Eau et d'Environnement, Département de Chimie, Faculté des Sciences Ain Chock, \\ Université Hassan II, Km 8, BP 5366, Maarif, Casablanca, Maroc. \\ ${ }^{2}$ Institut National de la Recherche Scientifique (INRS-ETE), Université de Québec, 490, Rue de la couronne, \\ QC J1K 9A9, Québec, Canada. \\ ${ }^{3}$ Ecole Hassania des Travaux Public, Km 7, Route d'El Jadida, Casablanca, Maroc. \\ ${ }^{*}$ Auteur correspondant, E-mail : zidanefatiha@hotmail.com; Tel : +212661330342
}

\section{RESUME}

L'industrie textile consomme une grande quantité d'eau, générant ainsi des rejets liquides à charge élevée en divers types de polluants. Ces derniers produisent des effets nocifs sur l'environnement. Ce travail s'intéresse à la dépollution d'un rejet d'une industrie textile, par addition d'un coagulant synthétique à base de fer et d'aluminium, préparé par le procédé d'électrocoagulation. Les résultats ont montré que le traitement de ce rejet par ce coagulant, a permis d'atteindre un rendement d'élimination des matières colorantes de $83.37 \%$ en terme de demande chimique en oxygène (DCO).

(C) 2011 International Formulae Group. All rights reserved.

Mots clés: traitement, hydroxyde, coagulation, demande chimique en oxygène.

\section{INTRODUCTION}

La présence de colorants dans les effluents industriels constitue un problème important dans plusieurs pays. Les industries qui rejettent de tels composés sont principalement les industries textiles et les industries de pâtes à papiers. La décharge de tels effluents dans le milieu récepteur cause une demande excessive en oxygène et ceux-ci doivent, par conséquent, être traités avant tout rejet dans l'environnement.

L'industrie de textile au Maroc est considérée comme l'un des principaux secteurs sur le plan économique et social, avec1800 entreprises, réparties sur l'ensemble $\mathrm{du}$ territoire nationale et emploie plus de 200000 personnes, c'est-à-dire $39 \%$ de l'effectif industriel du pays. L'industrie textile est de même, le meilleur exportateur des industries de transformation, avec près de $38 \%$ ce qui correspond à 25,5 milliards de dirhams de chiffre d'affaires à l'exportation d'après les statistiques de 1999 (Aitamalik et al., 2001).

La position géographique du Royaume, sa proximité de l'Union Européenne à michemin entre l'Europe et l'Afrique, ont permis à ce dernier de jouer un rôle important dans l'industrie du textile au niveau mondial $\left(6^{\text {ème }}\right.$ exportateur à l'échelle européenne). 
Ces activités de textile, génèrent des rejets chargés non seulement en colorants, mais aussi en d'autres substances toxiques, ce qui engendre une détérioration environnementale sans équivoque, et explique les multiples efforts déployés par les chercheurs, à travers le monde, pour mettre fin à ce danger qui menace notre milieu.

Pour cette raison, des recherches sur des nouvelles méthodes ont été effectuées, citons par exemple: des procédés biologiques utilisés pour le traitement des eaux usées municipales et qui se sont avérés inefficaces pour l'élimination des colorants (Robinson et al., 2001 ; Lazaridis et al., 2003;Walker et al., 2003).

Parmis ces nouvelles méthodes, citons aussi, les procèdes électrochimiques et chimiques, telles que, l'électrocoagulation et l'adsorption qui se sont avérés plus efficaces, pour l'élimination de ces colorants (Bauer, 2001 ; Dogan et al., 2003; Dali et al., 2006 ; Dos Suntos, 2007 ; Zuo et al., 2008 ; Zidane et al., 2008 ; Bennajah et al., 2009 ; Zongo et al., 2009).

Le présent travail, se divise en deux étapes:

La première consiste en une caractérisation chimique du rejet à traiter.

La deuxième consiste en un essai de dépollution à l'aide des coagulants à base de fer/aluminium préparés par électrocoagulation, et ceci afin de tester l'efficacité d'adsorption de ces derniers par la mesure des paramètres suivants: $\mathrm{pH}$, DCO et l'absorbance.

\section{MATERIEL ET METHODES}

La Figure 1 montre que l'eau d'alimentation de cette société de textile provient d'un réseau urbain, et alimenté par l'atelier de teinture, qui comporte sept machines (trois de lavages et quatre de teintures).

Cet atelier utilise différents types de colorants. Les effluents sortant des différents postes sont récupérés dans un bassin rectangulaire. Ce bassin est alimenté par des eaux de puits pour diluer les effluents venant de la teinture. Ainsi, les effluents dilués sont utilisés pour le lavage des cylindres des Storks utilisés dans l'atelier d'impression, ce qui va causer une augmentation de la charge polluante due aux colorants et pigments utilisés dans l'impression.

Ces rejets, à traiter, sont acheminés avec ceux de la cuisine et de la préparation des colorants vers un bassin de collecte et de traitement.

\section{Caractérisation chimique du rejet à traiter}

Les mesures de la Demande Chimique en Oxygène (DCO), de la Demande Biochimique en Oxygène $\left(\mathrm{DBO}_{5}\right)$, des Matières En Suspension (MES) et du potentiel Hydrogène $(\mathrm{pH})$ ont été effectuées par $\mathrm{La}$ LYDEC (Lyonnaise des Eaux de Casablanca), durant les années 2000, 2007, 2009, 2010 (Projet GEM ,1995).

\section{Traitement de rejet par adsorption sur des coagulants à base de fer et d'aluminium}

La dépollution du rejet globale, issu des différentes étapes de fabrication dans l'industrie textile, a été faite par l'addition d'un coagulant synthétique à base de fer et d'aluminium nommé (AF) préparé préalablement par électrocoagulation en utilisant une cellule électrolytique en pyrex dans laquelle a été introduite une solution de $5 \mathrm{~L}$ de $\mathrm{NaCl}$ de concentration $10^{-2} \mathrm{M}$ préparée avec de l'eau de robinet.

L'eau du robinet a été utilisée faisant référence, à celle qui est généralement utilisée dans l'industrie. La cellule électrolytique utilisée dans le présent travail est munie de quatre électrodes (deux d'aluminium et deux de fer) de dimensions $15 \mathrm{~cm} \times 15 \mathrm{~cm} \times 0.1 \mathrm{~cm}$. Deux d'entre elles sont connectées à la borne négative d'un générateur de courant continu (DC, AL, 823 ELC) et fonctionnent en cathodes. Deux autres à la cellule positive de celui-ci et jouent le rôle d'anode. Le potentiel est maintenu constant à $12 \mathrm{~V}$ durant $24 \mathrm{~h}$ à température ambiante. La distance inter électrode est de $4 \mathrm{~cm}$ (zidane et al., 2008).

Un volume de $500 \mathrm{ml}$ du rejet à traiter a été mis en contact avec des masses croissantes de $2.5 \mathrm{~g}$ à $12.5 \mathrm{~g}$ du coagulant 
(AF) préparé préalablement par électrocoagulation, et qui est composé principalement de $39 \%$ d'aluminium et de $20 \%$ de fer en pourcentage massique.

Après un temps de réaction de 5 min à température ambiante et sous agitation magnétique et un temps de décantation de 12 $\mathrm{h}$, des prélèvements ont été effectués afin de déterminer le $\mathrm{pH}$ à l'aide d'un $\mathrm{pH}$ mètre (Fisher Acument model 915 équipé d'une double jonction Cole palmer-électrode avec $\mathrm{Ag} / \mathrm{AgCl}$ cellule de référence), la DCO et l'absorbance ont été mesurées selon la méthode 5220D (Alpha et al., 1999) avec une courbe standard $(0-1000 \mathrm{mg} / \mathrm{L})$ à l'aide d'un spectromètre UV-visible (Varian modèle de Cary 50, Mississauga, Ontario, Canada).

\section{RESULTATS}

Caractérisation chimique du rejet traité Evolution du pH, $\mathrm{DCO}, \mathrm{DBO}_{5}$ et $\mathrm{MES}$

Les Figures 2, 3, 4 et 5 représentent respectivement l'évolution du $\mathrm{pH}$, de la DCO, de la $\mathrm{DBO}_{5}$ et des MES.

D'après la Figure 2, on constate que le pH diminue de la valeur 10,3 en 2000 jusqu'à 6,54 en 2010.

La DCO fluctue entre $929 \mathrm{mg} / \mathrm{L}$ et 8717 mg/L (Figure 3) à travers les années. Elle dépasse de loin la limite autorisée par la norme Marocaine $(500 \mathrm{mg} / \mathrm{L})$ pour les rejets directs (Benyakhlef et al., 2007) malgré la nette diminution de la DCO en 2010 (2,5 g/L) due à la dilution des rejets par la société avant le déversement de ce dernier dans le réseau. Rappelons qu'en 2000 la DCO est faible vu que la société venait de démarrer les activités de l'atelier de teinture.

Pour la $\mathrm{DBO}_{5}$, on remarque une fluctuation entre 125 et $925 \mathrm{mg} / \mathrm{L}$, aussi, pour la même période de 2000 à 2010 (Figure 4). Elle atteint $940 \mathrm{mg} / \mathrm{L}$, en 2007, et dépasse la norme Marocaine autorisée pour le rejet direct (100 mg/L) (Benyakhlef et al., 2007). La Figure 5 montre que Les matières en suspension varient, elles aussi, à travers les années, avec une diminution en 2010, qui est due à l'installation par la société d'un bassin de collecte et leur dilution fait diminuer les MES.
Pour avoir une estimation de la biodégradabilité de la matière organique d'un effluent, un calcul du rapport $\mathrm{DCO} / \mathrm{DBO}_{5}$ a été déterminé (Tableau 1).

D'après les résultats du Tableau 1, et sachant que si (El Guamri et al., 2006) :

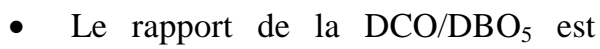
inférieur à 2, l'effluent est facilement biodégradable.

- Le rapport de la $\mathrm{DCO} / \mathrm{DBO}_{5}$ entre 2 et 3 , l'effluent est biodégradable avec des souches sélectionnées.

- Le rapport de la $\mathrm{DCO} / \mathrm{DBO}_{5}$ est supérieur à 3, l'effluent n'est pas biodégradable.

Pour le rejet étudié dans le présent travail, le rapport $\mathrm{DCO} / \mathrm{DBO}_{5}$ est supérieur à 3 au cour des années, ce qui montre que l'effluent n'est pas biodégradable, à l'exception de l'année 2010 qui est due à la dilution des effluents rejetés.

Il faut toutefois remarquer que la concentration des effluents reste élevée, ce qui risque d'inhiber l'activité biologique.

Traitement du rejet par adsorption sur le coagulant du mélange $\mathrm{Fe} / \mathrm{Al}$ synthétique préparé par électrocoagulation

Evolution du pH en fonction de la concentration $d u$ coagulant synthétique ajouté

Sur la Figure 6, est représentée l'évolution du $\mathrm{pH}$ en fonction des masses ajoutées des coagulants. D'après la figure, on constate que le $\mathrm{pH}$ diminue légèrement, au fur et à mesure que la concentration du coagulant synthétique à base $\mathrm{Fe} / \mathrm{Al}$ augmente jusqu'a une valeur de $\mathrm{pH}=7$.

Rendement d'élimination en DCO en fonction de la concentration du coagulant synthétique

Les résultats des mesures effectuées sont présentés sur la Figure 7. D'après cette dernière, on remarque que le rendement d'élimination en DCO augmente au fur et à mesure que la concentration du coagulant à base du mélange $\mathrm{Fe} / \mathrm{Al}$ augmente. On constate aussi que le meilleur rendement obtenu est de $83.37 \%$ pour une concentration de $20 \mathrm{~g} / \mathrm{L}$ de coagulant ajouté. 
Rendement de décoloration en fonction de la concentration du coagulant synthétique ajouté

La Figure 8 présente les rendements de décoloration en fonction du coagulant ajouté. D'après cette dernière, on observe que l'absorbance diminue au fur et à mesure que la concentration du coagulant à base $\mathrm{Fe} / \mathrm{Al}$ augmente. La concentration optimale du coagulant ajouté est de $20 \mathrm{~g} / \mathrm{L}$.

L'utilisation du coagulant à base $\mathrm{Fe} / \mathrm{Al}$ synthétisé au laboratoire peut se révéler intéressante quant à sa facilité d'utilisation.
La concentration de coagulant à base de Fe/Al peut sembler, par ailleurs, assez élevée puisqu'une concentration de $20 \mathrm{~g} / \mathrm{L}$ est nécessaire pour atteindre une décoloration presque totale du rejet coloré. Celle-ci n'aura néanmoins aucun impact néfaste sur la qualité de l'eau rejetée, puisque la zone de $\mathrm{pH}$ correspondant au traitement est celle de prédominance des hydroxydes, située entre 5 et 7,2 pour $\mathrm{Al}(\mathrm{OH})_{3}$ et 5,5 et 8,3 pour $\mathrm{Fe}(\mathrm{OH})_{3}$, pour laquelle les coagulants sont très stables en solution et existent sous forme solide.

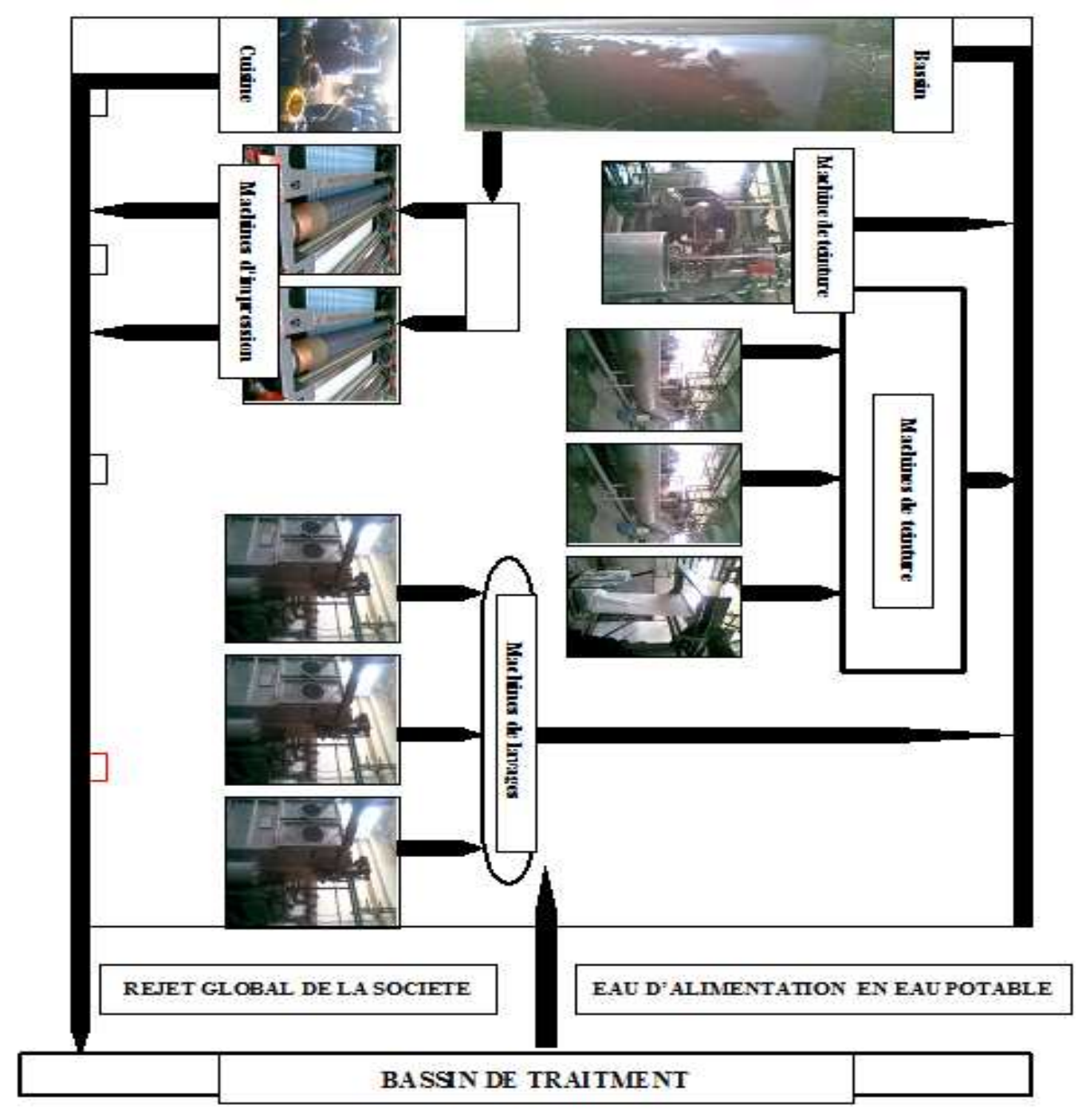

Figure 1 : Le circuit de l'eau dans la société de textile. 


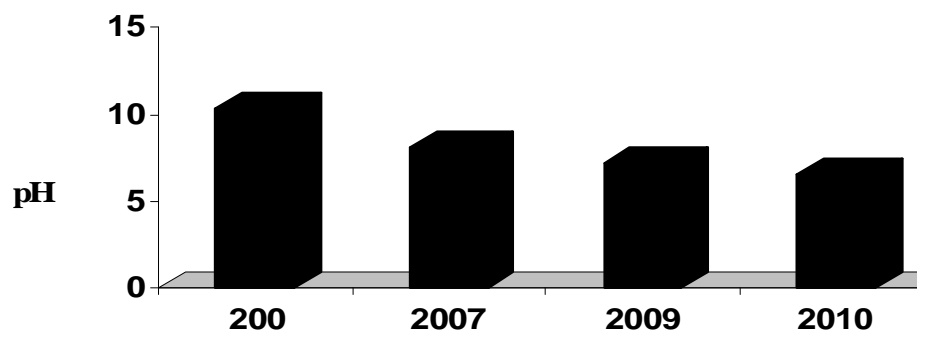

Années d'analyse

Figure 2 : Evolution de pH en fonction des années.

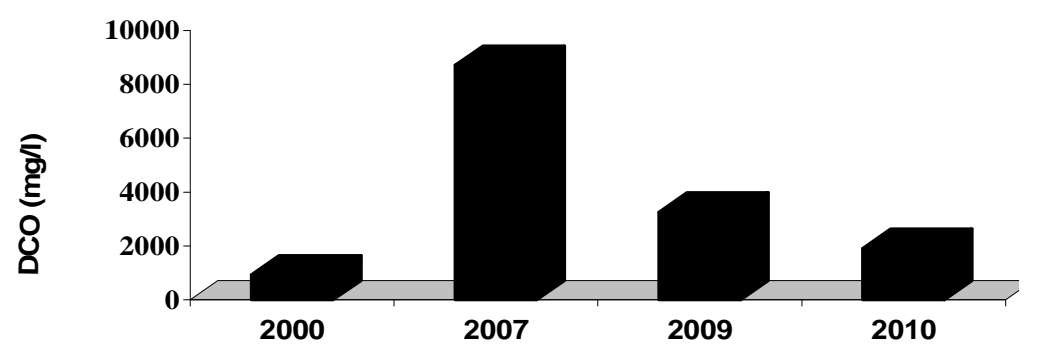

Années d'analyse

Figure 3 : Evolution de la Demande Chimique en Oxygène (DCO) en fonction des années.

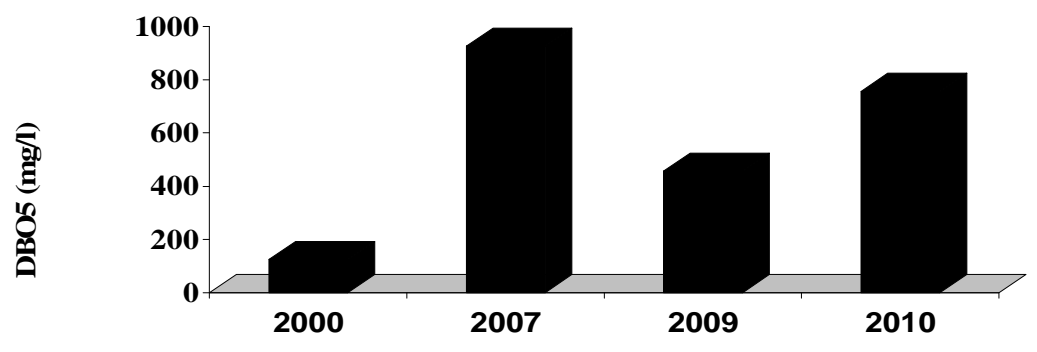

Années d'analyse

Figure 4 : Evolution de la Demande Biochimique en Oxygène $\left(\mathrm{DBO}_{5}\right)$ en fonction des années. 


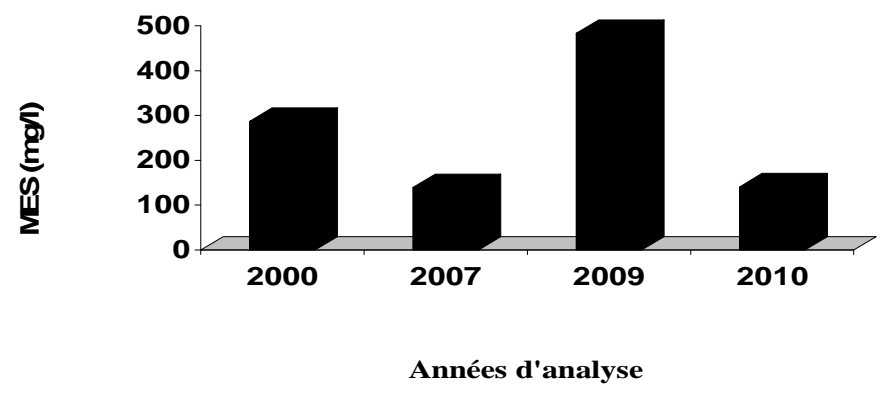

Figure 5 : Evolution des Matières en Suspension (MES) en fonction des années.

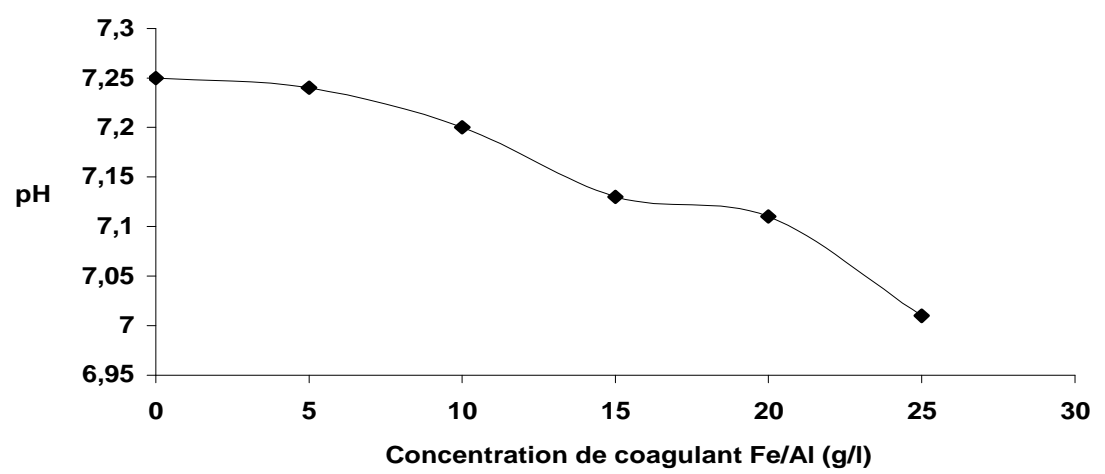

Figure 6 : Évolution de $\mathrm{pH}$ en fonction de concentration du coagulant synthétique à base de fer et aluminium.

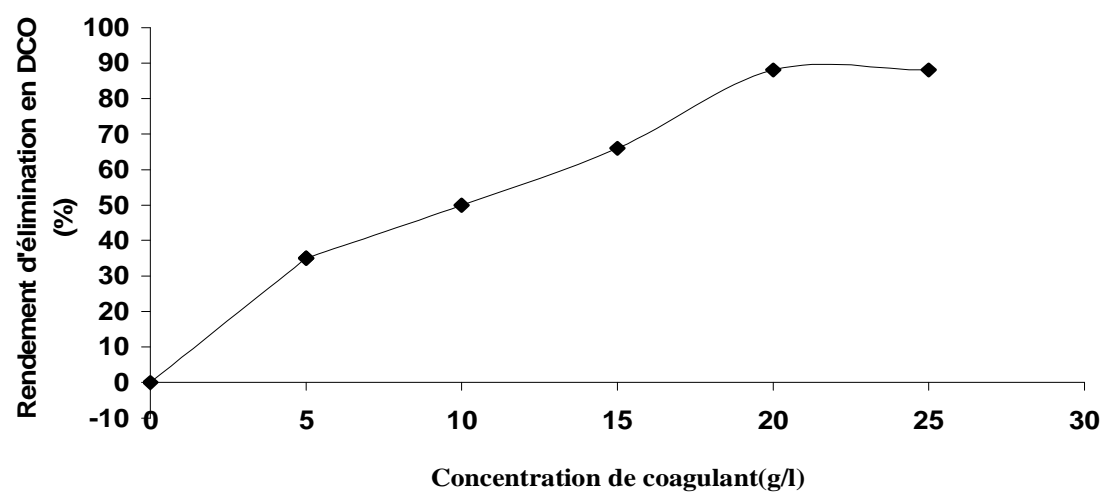

Figure 7: Rendement d'élimination en DCO en fonction de la concentration du coagulant à base de fer et aluminium. 


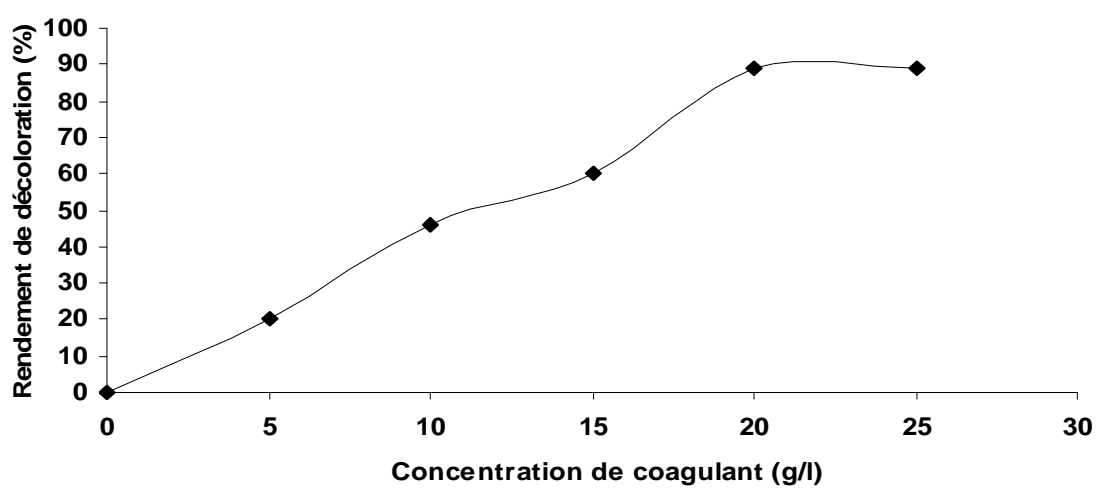

Figure 8: Rendement de décoloration en fonction de la concentration du coagulant synthétique à base de fer et aluminium.

Tableau 1: rapport de biodégradabilité des effluents rejetés par la société.

\begin{tabular}{lccc}
\hline ANNEE & DCO $(\mathbf{m g} / \mathbf{l})$ & DBO $_{\mathbf{5}}(\mathbf{m g} / \mathbf{l})$ & DCO/DBO $_{\mathbf{5}}$ \\
\hline 2000 & 929 & 125 & 7.43 \\
2007 & 8717 & 926 & 9.71 \\
2009 & 3264 & 456 & 7.15 \\
2010 & 1905 & 756 & 2.51 \\
\hline
\end{tabular}

\section{DISCUSSION}

Caractérisation chimique du rejet traité

L'évolution du $\mathrm{pH}$, de la $\mathrm{DCO}, \mathrm{DBO}_{5}$ et MES sur les quatre années est due à l'importance des activités telles que le désencollage, le mercerisage, le blanchiment, la teinture et l'impression utilisant différentes matières telles que les colorants, les adjuvants, les épaississants et les fixateurs, qui sont présentes dans les différentes étapes du processus de fabrication dans cette société.

Traitement du rejet par adsorption sur le coagulant du mélange $\mathrm{Fe} / \mathrm{Al}$ synthétique préparé par électrocoagulation Evolution de pH en fonction de la concentration du coagulant synthétique ajouté

Dans le cas du traitement du rejet global, et d'après l'ajout de différentes masses du coagulant synthétique à base $\mathrm{Fe} / \mathrm{Al}$, le $\mathrm{pH}$ atteint une valeur de 7 (Figure 6), pouvant expliquer l'élimination des polluants de ce rejet et l'obtention d'une eau claire et limpide (Zidane et al., 2008).

Rendement de DCO en fonction de la concentration du coagulant synthétique ajouté

Le rendement d'élimination en DCO augmente au fur et à mesure que la concentration du coagulant à base du mélange $\mathrm{Fe} / \mathrm{Al}$ augmente (Figure 7), ceci peut être expliqué par l'élimination d'une partie des substances colloïdales et dissoutes des effluents traités probablement par adsorption sur les flocs d'hydroxyde d'aluminium et de fer qui se forment au $\mathrm{pH}$ des effluents.

Rendement de décoloration en fonction de la concentration de coagulant synthétique ajouté

L'absorbance diminue au fur et à mesure que la concentration du coagulant à 
base $\mathrm{Fe} / \mathrm{Al}$ augmente (Figure 8), ce qui peut être due à la décoloration de ce rejet. Le coagulant étant constitué essentiellement d'hydroxydes de fer et d'aluminium (Zidane et al., 2008) est très stable à des $\mathrm{pH}$ proches de 7 et donc très peu susceptible de se dissoudre autrement dit d'exister sous forme ionique et donc polluante. Celui-ci se trouvant ainsi en majorité constitué d'hydroxydes, représente une surface possédant une grande capacité d'adsorption expliquant les rendements élevés d'élimination des matières colorantes.

\section{Conclusion}

Cette étude a permis la dépollution d'un rejet complexe issu des différentes activités de la société de textile par un coagulant à base de fer/aluminium préparé par électrocoagulation. La variation des paramètres tels que le $\mathrm{pH}$, la DCO, la $\mathrm{DBO}_{5}$ et des MES observée aux cours des quatre années est probablement due à l'importance des activités de la production ainsi qu'aux matières utilisées, présentes dans les différentes étapes du processus de fabrication au sein de la société.

Le rendement d'élimination des matières organiques en DCO et de décoloration en absorbance est important. Il peut être dû à l'élimination d'une partie des substances colloïdales et dissoutes des effluents traités par adsorption sur les flocs d'hydroxyde d'aluminium et de fer formés au pH des effluents et à la capacité d'adsorption des coagulants .

Les résultats de décoloration et d'élimination de la DCO sont influencés par les différentes activités de la société de textile ainsi que par les concentrations de différentes matières ajoutées aux cours de la fabrication des vêtements.

\section{REFERENCES}

Aitamalik S, Allali B. 2001. Le secteur textiles-habillement marocain face aux défis de la modalisation, institut supérieur de commerce et d'administration des entreprises.

Apha, Awwa , Wpcf . 1999. Standars Methods for Examination of Water and Wastewaters (20 é éd). American Public Health Association, American water works association et Water Pollution Control Federation: Washintgon D.C.

Bauer C, Jacques P, Kalt A. 2001. Photooxidation of an azo dye induced by visible light incident on the surface of TiO2. J. Photochem. Photobiol. A: chem., 140: 87-92.

Bennajah B, Gourich B, Essadki A.H, Vial $\mathrm{CH}$, Delmas H. 2009. Defluridation of Morocco drinking water by electrocoagulation/ electroflotation in an electrochemical external-loop airlift reactor.Chemical Energineering Journal, 148: $122-131$.

Benyakhlef F, Naji S, Belghyti D. 2007. Caractérisation des rejets liquides d'une conserverie des poissons. Bull. Soc. Pharm., 146: 225-234.

Dali ZY, Bouabdassalam H, Bettar N. 2006. Elimination des composés organiques par des argiles locales. C. R. Chimie, 9: 1295-1300.

Dogan M, Alkan M. 2003. removol of methyl violet from aqueous solution by perlite. Colloid Interface Sci., 267: 32-41.

Dos Santos AB, Cervantes FJ, Van Lier JB. 2007. Review paper on current technologies for decolourisation of textile wastewaters: Perspectives for anaerobic biotechnology. Bioresource Technology, 98: 2369-2385.

El Guamri Y, Belghyti D. 2006. Etude de la qualité physico-chimique des eaux usées brutes de la commune urbaine de Saknia, rejetées dans le lac Fouarat (Kénitra, Maroc). Journal Africain des Sciences de l'Environnement, 1: 53-60.

Lazaridis NK, Karapantsios TD, Georgantas D. 2003. Kinetic analysis for the removal of a reactive dye from aqueous solution 
onto hydrotalcite by adsorption. Water Res., 37: 3023-3033.

Projet GEM.1995. Gestion de l'énergie dans les entreprises marocaines, manuel sur la prévention de la pollution dans l'entreprise. Projet N²98-0365.08.

Robinson T, Mucmullan G, Marchant R, Nigam P. 2001. Remediation of dyes in textile effluent: A critical review on current treatment technologies with a proposed alternative. Biores. Technol., 77: 247-255.

Zidane F, Drogui D, Lekhlif B, Bensaid J, Blais JF, Belcadi S, El kacemi K. 2008. Decolourization of dye-containing effluent using mineral composé synthétiques produced by electrocoagulation. Journal of Hazardous Materials, 155: 153-163.
Zongo I, Maiga AH, Wéthé J, Valentine G, Leclerc JP, Paternotte G, Lapicque F. 2009. Electrocoagulation for the treatment of textile wastewater with $\mathrm{Al}$ or Fe electodes compared variation of COD levels, turbidity and absorbance. Journal of Hazardous Material, 169: 70-76.

Zuo Q, Chen X, Li W, Chen G. 2008. Combined electocoagulation and electroflotation for removal of fluoride from drinking water. Journal of Hazardous Material, 159: 452-457.

Walker GM, Hansen L, Hanna JA, Allen SJ. 2003. Kinetics of a reactive dye adsorption onto dolomitic sorbents. Water Res., 37 : 2081-2089. 